\title{
Composition fluctuations before dewetting in polystyrene/poly(vinyl methyl ether) blend thin films
}

$\operatorname{AUTHOR}(\mathrm{S}):$

Ogawa, Hiroki; Kanaya, Toshiji; Nshida, Koji; Matsuba, Go

\section{CITATION:}

Ogawa, Hiroki ... [et al]. Composition fluctuations before dewetting in

polystyrene/poly(vinyl methyl ether) blend thin films. Polymer 2008, 49(10): 2553-2559

\section{ISSUE DATE:}

2008-05-13

URL:

http://hdl.handle.net/2433/93442

\section{RIGHT:}

Copyright (c) 2008 Elsevier; This is not the published version. Please cite only the published version.; この論文は出版社版でありません。引用の 際には出版社版をご確認ご利用ください。 


\title{
Composition Fluctuations before Dewetting in
}

\section{Polystyrene/Poly(vinyl methyl ether) Blend Thin Films}

\author{
Hiroki Ogawa, Toshiji Kanaya, Koji Nshida and Go Matsuba
}

Institute for Chemical Research, Kyoto University, Kyoto, Japan

$\begin{array}{ll}\text { corresponding author: } & \text { T. Kanaya } \\ \text { Postal address: } & \text { Institute for Chemical Research, Kyoto University } \\ & \text { Uji, Kyoto-fu, 611-0011 } \\ & +81-774-38-3140,+81-774-38-3146 \\ \text { Tel. \& Fax.: } & \text { kanaya@scl.kyoto-u.ac.jp }\end{array}$




\section{abstract}

We report structure development in blend thin films of deuterated polystyrene (dPS) and poly(vinyl methyl ether) (PVME) below $\sim 200 \mathrm{~nm}$ in two phase region during the incubation period before dewetting using neutron reflectivity (NR) and atomic force microscope (AFM). As was predicted by the former optical microscope (OM) and small-angle light scattering (LS) measurements on blend thin films of protonated PS (PS) and PVME [Polymer, 40, 254-22 (2008)], the NR results clearly showed that the tri-layer structure consisting of the surface PVME layer, the middle blend layer and the bottom PVME layer was formed in the one phase region. After the temperature jump into the two phase region, it was found that the phase separation of the middle blend layer proceeded in the depth direction during the incubation period before dewetting, suggesting that the dewetting was induced by the composition fluctuations during the incubation period. 


\section{Introduction}

Extensive studies have been performed on polymer thin films including single component and multi component systems [1-4] because properties of polymer thin films are directly related to many important phenomena such as coating, adhesive, surface friction, lubricants, and dielectric layer. The studies on polystyrene (PS) thin films on Si substrates have revealed that glass transition temperature $T_{\mathrm{g}}$ decreased with film thickness $d$ below about $40 \mathrm{~nm}$ [5-9], but increased with decreasing film thickness $d$ for poly(methyl methacrylate) (PMMA) $[5,6]$, showing the importance of interactions between polymers and substrates. Recently, dewetting and phase separation of polymer blend thin films are also of great interest because of technological applications as well as fundamental investigations. The dewetting of the phase separated polymer blend thin film limits the industrial applications because they require stable, homogeneous and uniform thin films. This motivated the fundamental studies of polymer blend thin films very much to reveal the dewetting mechanism and control the morphology [4] for blend thin films of poly( $p$-methylstyrene) (PpMS) and deuterated polystyrene (dPS) [10], poly (styrene-ran-acrylonitrile) (SAN) and deuterated poly (methyl methacrylate) (dPMMA) [11-15], and PS and poly (vinyl methyl ether) (PVME) [16-19] in various film thickness ranges.

As for the dewetting Composto et al. [13, 14] have proposed the capillary fluctuation mechanism for the blend thin films of SAN and dPMMA $\left(R_{\mathrm{g}}<d<150 R_{\mathrm{g}}\right.$, $R_{\mathrm{g}}$ being radius of gyration of a polymer). On the other hand, Chung et al. [20] have recently shown that the phase separation in thin films of SAN and dPMMA $(d=550$ $\mathrm{nm}$ ) drove the dewetting and the capillary fluctuations failed to explain the dewetting. Liao et al. [15] have experimentally demonstrated that the composition fluctuation 
mechanism [21-24] induced the dewetting in thermodynamically stable ultrathin blend films of SAN and dPMMA in one phase region. Thus, the dewetting mechanism in blend thin films is still a controversial problem.

In a previous paper [25], therefore, we investigated morphology and kinetics of phase separation as well as dewetting in blend thin films of PS/PVME on glass substrate in two phase region as a function of film thickness in a wide thickness $d$ range from 65 $\mu \mathrm{m}$ to $42 \mathrm{~nm}\left(\sim 2.5 R_{\mathrm{g}}\right)$ to elucidate the relation between phase separation and dewetting. We found that the dewetting occurred in a spinodal decomposition (SD) mechanism after a long incubation time in a thickness range below $\sim 200 \mathrm{~nm}$. The observations indicated a layered structure was formed in the incubation time before the dewetting although direct evidences for the layered structure were not obtained in the previous work. In order to confirm the prediction of the layered structure we have mainly studied structural development in the depth direction during the incubation period before the dewetting for thin blend films of deuterated PS (dPS) ad PVME in a thickness range below $\sim 200 \mathrm{~nm}$ using neutron reflectivity.

\section{Experiment}

Polystyrene (PS) and poly(vinyl methyl ether) (PVME) used in this study have weight-average molecular weights $M_{\mathrm{w}}=280,000$ and 90,000, and the molecular weight distributions in terms of $M_{\mathrm{w}} / M_{\mathrm{n}}=3.01$ and 1.88 , respectively, where $M_{\mathrm{n}}$ is numberaverage molecular weight. We also used deuterated polystyrene (dPS) with weightaverage molecular weight $M_{\mathrm{w}}=280,000$ and the molecular weight distribution $M_{\mathrm{w}} / M_{\mathrm{n}}=$ 1.08 for neutron reflectivity measurements. Both of PS (dPS) and PVME were purchased from Scientific Polymer Products, Inc. PS (dPS) and PVME were purified 
by precipitating the toluene solutions into excess methanol and n-heptane several times, respectively, and dried in vacuum at room temperature for $72 \mathrm{~h}$. Films below and above $1 \mu \mathrm{m}$ were prepared by spin-coating and casting the toluene solution of PS and PVME, respectively, on a cleaned glass substrate after filtering with $2 \mu \mathrm{m}$ pore size membrane at room temperature for optical microscope $(\mathrm{OM})$, atomic force microscope (AFM) and small-angle light scattering (LS). The films were then annealed at $60{ }^{\circ} \mathrm{C}$ for $24 \mathrm{~h}$ after drying in vacuum at room temperature for $24 \mathrm{~h}$. Thickness of polymer film was controlled by varying the polymer concentration in solution and confirmed with ellipsometer measurements. Blend films of dPS and PVME were prepared on a cleaned Si wafer with native oxide layer in the same manner as for the PS and PVME films.

The phase diagram of PS and PVME has been determined in the previous work [25], which is a lower critical solution temperature (LCST) type phase diagram. The critical weight fraction of PS is 0.3 and the critical temperature is $104.5{ }^{\circ} \mathrm{C}$. All the measurements were done on the blend samples with the critical concentration. The glass transition temperature $T_{\mathrm{g}}$ of the bulk blend with the critical concentration $\left(\phi_{\mathrm{PS}}=\right.$ 0.3 ) is $-20{ }^{\circ} \mathrm{C}$. The coexisting compositions are nearly pure components at $115{ }^{\circ} \mathrm{C}$, which were estimated by extrapolating the phase diagram.

Small-angle light scattering (LS) measurements were carried out using home-made apparatus with confocal collimation, which enables us to access a very low $q$ range down to $0.1 \mu^{-1}$ with extremely low background. Two dimensional scattering intensities were accumulated every 5 min after temperature jump from one phase region to two phase region $\left(=115^{\circ} \mathrm{C}\right)$ with a heating rate of $36^{\circ} \mathrm{C} / \mathrm{min}$. Details of the $\mathrm{LS}$ instrument were reported elsewhere [26]. Optical microscope (OM) and confocal laser 
scanning microscope (CLSM) measurements were also done after temperature jump from one phase region to two phase region $\left(=115{ }^{\circ} \mathrm{C}\right)$ using OLYMPUS BX50 equipped with a CCD camera and LASER TECH. ILM15, respectively. Atomic force microscope (AFM) measurements were performed at room temperature after quenching the sample from two phase region $\left(=115^{\circ} \mathrm{C}\right)$ using JEOL JSPM-4200 to examine the surface morphology of the films.

Neutron reflectivity measurements were done on MINE-II reflectometer at JRR-3 reactor in Tokai [27]. Incident neutron wavelength was $0.88 \mathrm{~nm}$ and the $q$ range covered in the present measurements was 0.07 to $1.0 \mathrm{~nm}^{-1}$. The measurements was conducted at room temperature after quenching the sample from two phase region $\left(=115^{\circ} \mathrm{C}\right)$. We analyzed the observed reflectivity data using the Paratt32 program in Hahn-Meitner-Institute [28], which is based on a recursion formula derived by Parratt [29] to calculate reflectivity from successive interfaces and modified to include effects of interfacial roughness.

\section{Results and Discussion}

We first recall the previous results [25] to clarify the aim of this work. We have studied the phase separation and dewetting processes of blend thin films of PS and PVME with the critical concentration in a wide thickness range of $42 \mathrm{~nm}$ to $65 \mu \mathrm{m}$ after a temperature jump to $115^{\circ} \mathrm{C}$ in the two phase region from the one phase region using OM, AFM and LS. We found that both of the phase separation and dewetting processes depended on the film thickness, and were classified into four thickness regions. In order to explain the characteristic features in the four regions the typical $\mathrm{OM}$ and AFM images at 90 min after the temperature jump are shown in Figures 1(a) 
and (b), respectively, and the time evolutions of the LS profiles are shown in Figure 1(c) for the four regions. In the first region above $\sim 15 \mu \mathrm{m}$ (Region I), the spinodal decomposition (SD) type phase separation is observed in the OM image, which is also confirmed from the time evolution of LS intensity. In this region no dewetting is observed in the AFM image, and hence this region can be regarded as bulk region. In the second region between $\sim 15$ and $\sim 1 \mu$ m (Region II) the SD type phase separation proceeds in the early stage similar to Region I while the characteristic wavelength decreases with the film thickness due to the confinement effect of film thickness in this region. In the late stage the dewetting is induced by the phase separation. In the third region between $\sim 1 \mu \mathrm{m}$ and $\sim 200 \mathrm{~nm}$ (Region III) the dewetting is observed even in the early stage. The dewetting morphology is very irregular as seen in Figures 1(a) and (b) and no definite characteristic wavelength is observed in the LS profile. It is expected that the irregular morphology is induced by mixing up the characteristic wavelengths of the phase separation and the dewetting. In the forth region below $\sim 200 \mathrm{~nm}$ (Region IV) the dewetting occurs after a long incubation time with a characteristic wavelength, giving a peak in the LS profile as seen in Figure 1(c). Note that the LS profiles in Figure 1(c) are shown until $60 \mathrm{~min}$ for Region IV. Generally speaking, the SD type mechanism occurs in an unstable region $[30,31]$, meaning that no incubation time is required. However, the long incubation time is observed before the dewetting, suggesting that some structure formation occurs before the onset of the SD type dewetting. Therefore, we examined structure formation in the incubation period before dewetting to discuss the dewetting mechanism in Region IV below $\sim 200 \mathrm{~nm}$.

In Figure 2, time evolution of the $\mathrm{OM}$ images is shown for the $98 \mathrm{~nm}$ film after temperature jump to $115^{\circ} \mathrm{C}$ to see what happens in the incubation period. Nothing is 
observed in the $\mathrm{OM}$ images before $\sim 20$ min within the present $\mathrm{OM}$ contrast and the spatial resolution while very weak in-plane fluctuations are recognized after $\sim 20 \mathrm{~min}$ as seen in Figure 2. At $\sim 30$ min the SD type patterns becomes rather clear and grows in size with the annealing time. According to the changes in the OM images, we also observe the changes in the LS profiles. In Figure 3 we plot the time evolution of the LS intensity $I_{\max }$ and the peak position $q_{\max }$. The LS peak appears at 30 min after the temperature jump, corresponding to the appearance of the SD pattern in the OM image. It is noted that the LS intensity slightly increases until $\sim 30 \mathrm{~min}$ in the incubation period, which must be due to the very weak in-plane fluctuations observed in the OM image. After $\sim 30$ min the peak intensity $I_{\max }$ increases rapidly with the annealing time and the peak position shifts to lower $q$. It is clear from the AFM images in Figure 1(b) that this SD type pattern is caused by the dewetting.

As discussed in the previous paper [25] we expect that some layered structure is formed during the incubation period, and triggers the dewetting through the capillary fluctuations mechanism $[13,14]$ or the composition fluctuation one $[15,21-24]$. However, no direct evidence was provided for the layered structure. In order to confirm the layered structure we examined the composition fluctuations in the direction normal to the surface (the depth direction) before dewetting using neutron reflectivity (NR), which is one of the most powerful tools to study composition and/or density fluctuations in the depth direction.

We used a $\mathrm{Si}$ wafer as a substrate in the NR measurements instead of a glass substrate, which was used for OM, LS and AFM in the previous paper [25]. It is known that the dewetting is affected by interface interactions between polymer film and substrate. In addition we used deuterated polystyrene (dPS) in the NR experiments. 
It would be impossible to consider these two systems (PS/PVME on a glass substrate and $\mathrm{dPS}$ /PVME on a Si substrate) are identical. Therefore we first examined the dPS/PVME blend film $98 \mathrm{~nm}$ thick using confocal laser scanning microscope (CLSM). We found that the dewetting patterns were almost identical but the onset time was slower in the case of the Si substrate than the glass one. Exactly speaking, it is $60 \mathrm{~min}$ at $115{ }^{\circ} \mathrm{C}$ for the $\mathrm{Si}$ substrate and $30 \mathrm{~min}$ for the glass substrate. No other large differences were observed between the Si and glass substrates. However, the phase separation and dewetting are so sensitive to the substrates that we discuss the results on the dPS/PVME thin films on the Si substrate independent of the PS/PVME thin films on the glass substrate in this paper.

We performed NR measurements on the dPS/PVME blend films at room temperature in the one phase region before annealing at $115^{\circ} \mathrm{C}$ in the two phase region. The observed NR profiles are shown in Figure 4 for the 180, 98, 42 and $25 \mathrm{~nm}$ films. The well defined fringed patterns were observed in the profiles. The NR profiles were analyzed by fitting a single layer model on a Si substrate with a native oxide layer to the observed data, but good fitness was not obtained. Then, we tried bi-layer and tri-layer models consisting of surface PVME layer at the air, middle blend layer, and interface PVME layer at the Si substrate because the preferential interactions are considered between PVME and air [32-36] and between PVME and Si substrate $[17,18]$. In this model the surface roughness $\sigma_{\mathrm{NR} \text {,surf }}$ and the interface roughness $\sigma_{\mathrm{NR} \text {,inter }}$ were introduced by an error function (eq. (1)) 


$$
\operatorname{erf}(x)=\int_{x}^{\infty} \frac{1}{\sqrt{2 \pi} \sigma_{i}} \exp \left(-\frac{t^{2}}{2 \sigma_{i}^{2}}\right) d t \quad(i=\mathrm{NR}, \text { inter or NR,surf })
$$

It was found that the tri-layer model gave good agreements between the observed reflectivity and the calculated one as shown in Figure 4 . In inset of Figure 4 the scattering length density evaluated in the fit is shown for each film. The estimated surface and interface PVME layers are $\sim 4 \mathrm{~nm}$ and $\sim 5 \mathrm{~nm}$ thick and almost independent of the total film thickness within the experimental error. The surface roughness, the interface roughness between the surface PVME layer and the middle blend layer and the interface roughness between the middle blend layer and the bottom PVME layer are 3.5, 4.5 and $5 \mathrm{~nm}$ for the $180 \mathrm{~nm}$ film, respectively, and slightly decrease with the total film thickness. It is expected that the surface and interface PVME layer formation increase the dPS concentrations in the middle blend layer, especially in the thin films. Hence, we have evaluated the PS concentrations in the middle blend layer and found that the effect is negligible for the 180 and $98 \mathrm{~nm}$ films, but not negligible for the $42 \mathrm{~nm}$ and 25 nm films. In case of the $25 \mathrm{~nm}$ film the estimated dPS concentration $\phi_{\mathrm{PS}}$ in the middle blend layer is 0.47 . Therefore, the further experiments were performed on the $98 \mathrm{~nm}$ film. In any case the present results clearly show that the dPS/PVME blend thin films on the Si substrate have tri-layer structure even in the one phase region due to the preferential interactions.

In the next step we have examined the time evolution of the tri-layer structure in the $98 \mathrm{~nm}$ film during the incubation period before the dewetting. The film was annealed at $115^{\circ} \mathrm{C}$ in the two phase region for a given period, and quenched to room 
temperature in the one phase region. The NR measurements were performed on the quenched film. In Figure 5(a) the observed NR profiles are shown for various annealing periods. The fringed pattern is gradually smeared with the annealing time even in the incubation period ( $\sim 60 \mathrm{~min})$, suggesting that the surface roughness and/or the interface roughness increases with the annealing time before the dewetting. At 120 min the fringed pattern is completely smeared out, showing the film is dewetted. It is surprising that the fringed pattern with a long period was again observed at $360 \mathrm{~min}$, implying that a thin layer was formed on the surface of the substrate after the dewetting. This problem will be discussed later.

The NR profile calculated from the tri-layered model was fitted to the observed one to evaluate the thickness and the surface and interfacial roughness for each layer. The results of fits are shown by solid curves in Figure 5(a) and the depth profiles of the corresponding scattering length density are plotted in Figure 5(b). The interface roughness $\sigma_{\mathrm{NR} \text {,inter }}$ between the top PVME layer and the middle blend layer increases with the annealing time during the induction period, but the interface roughness between the bottom PVME layer and the middle blend layer stays almost constant $(\sim 5 \mathrm{~nm})$. The surface roughness $\sigma_{\mathrm{NR} \text {,surf }}$ of the top PVME layer seems to increase with the annealing time, but it is noted that the NR profile is not sensitive to the surface roughness of the top PVME layer because of the low scattering length density of PVME so that we do not discuss the surface roughness here. The former interface roughness $\sigma_{\mathrm{NR}, \text { inter }}$ are plotted as a function of the annealing time in Figure 6. It is evident that they increase even during the incubation period. Before going to the discussion on the time evolution of the roughness, we introduce the surface roughness $\sigma_{\mathrm{AFM} \text {,surf }}$ evaluated 
by the AFM measurements because the surface roughness $\sigma_{\mathrm{NR} \text {,surf }}$ estimated by the NR measurements includes large error.

The observed AFM images of the dPS/PVME film $98 \mathrm{~nm}$ thick are shown in Figure 7 for various annealing times in the incubation period. The surface is very smooth at the annealing time $=0 \mathrm{~min}$. As the annealing proceeds no significant changes were observed during the incubation period. In order to evaluate the surface roughness the AFM data was analyzed in the following way. The distribution of surface heights $h$ was calculated from the AFM data. An example is shown in Figure 8 for the $98 \mathrm{~nm}$ film annealed at $115^{\circ} \mathrm{C}$ for $5 \mathrm{~min}$. Assuming a Gaussian distribution of $h$, the standard deviation $\sigma_{\mathrm{AFM} \text {,surf }}$ was evaluated by fitting eq. (2) to the observed distribution.

$$
f(h)=\frac{1}{\sqrt{2 \pi} \sigma_{\mathrm{AFM}, \text { surf }}} \exp \left(-\frac{(h-\bar{h})^{2}}{2 \sigma_{\mathrm{AFM}, \text { surf }}^{2}}\right)
$$

where $\bar{h}$ is the average value of the height. The result of fit is shown by a solid curve in the figure. As seen in Figure 8 the distribution is well described by a Gaussian function in the incubation period. The evaluated surface roughness $\sigma_{\mathrm{AFM} \text {,surf }}$ is plotted in Figure 6 as a function of the annealing time in the incubation period to compare with the interface roughness $\sigma_{\mathrm{NR} \text {,iner }}$ evaluated by the NR measurements.

The AFM surface roughness $\sigma_{\mathrm{AFM} \text {,surf }}$ is almost independent of the annealing time. On the other hand, the interface roughness $\sigma_{\mathrm{NR} \text {,inter }}$ increases with the annealing time from the beginning of the incubation time. In the two phase region it is expected that the phase separation would occur in the middle blend layer into PVME-rich and dPS- 
rich phases. Nevertheless, no changes were observed during incubation period in the CLMS images, suggesting that the phase separation dose not proceed at least in the inplane direction. The increase of the NR interface roughness $\sigma_{\mathrm{NR} \text {,inter }}$ in the incubation period indicates that the phase separation proceeds in the depth direction, which must be induced and accelerated by the preferential interactions between PVME and air [35]. This phase separation in the depth direction does not occur coherently in the depth direction like the surface directed spinodal decomposition (SDSD) [37], but inhomogeneously in the in-plane direction [15].

In the following we will discuss the dewetting mechanism on the basis of the observations. In the previous paper [25] we considered two possibilities for the dewetting mechanism for the PS/PVME thin films in Region IV below $200 \mathrm{~nm}$ : the capillary fluctuation mechanism $[13,14]$ and the composition fluctuation mechanism $[15,21-24]$. The former mechanism was proposed by Composto et al. $[13,14]$ to explain the dewetting of deuterated poly(methyl methacrylate) (dPMMA) and poly(styrene-ran-crylonitrile) (SAN) in a thickness $d$ range of $R_{\mathrm{g}}<d<150 R_{\mathrm{g}} . \quad$ In order to check the mechanism we evaluated the capillary fluctuation for the interface between the surface PVME and the middle blend layer based on the theory by Shull et al. [38]. It was found that the fluctuation length was less than $3.3 \mathrm{~nm}$, which is much smaller than the interface roughness derived from the NR result in the incubation period, implying a less possibility of the capillary fluctuation mechanism.

The composition fluctuation mechanism was theoretically introduced by some researchers [21-24] and An and coworkers [15] applied this idea to explain the dewetting of thermodynamically stable blend ultrathin film of DPMMA and SAN with $d \sim R_{\mathrm{g}} . \quad$ In this mechanism polymers which have preferable interactions with the surface 
(or the substrate) diffuse to the surface (or the substrate) so as to create the composition gradient across the film. The diffusion could not occur homogeneously over the film surface to create the composition fluctuations in the mixture along the surface. When the amplitude of the fluctuations is large enough, the free surface is eventually destabilized, leading to the dewetting [21-24]. This mechanism is also possible in the two phase region. In the present experiment we observed tri-layer structure in the dPS/PVME blend thin film $98 \mathrm{~nm}$ thick. The film was stable in the one phase region and no dewetting occurs. After the temperature jump into the two phase region, however, we found that the interface roughness $\sigma_{\mathrm{NR} \text {,inter }}$ increases rapidly with the annealing time in the incubation period, showing that the composition fluctuations proceed in the depth direction with the annealing time, and the composition fluctuations may proceed inhomogeneously in the in-plane direction. These inhomogeneous composition fluctuations must induce the dewetting when the amplitude is enough large that the surface is destabilized. Thus, the present observations support the composition fluctuation induced dewetting mechanism for the dPS/PVME blend film below 200 nm.

Finally we would like to comment on the fringed pattern observed in the NR profile at $360 \mathrm{~min}$ after the temperature jump in Figure 5(a). The fringed pattern with a long period suggests that a very thin PVME layer is formed in a very late stage after the dewetting. The thickness evaluated by fitting is $\sim 10 \mathrm{~nm}$, which is shown in the scattering length density profile in Figure 5(b). Why does such a thin film is formed after the dewetting? In the incubation period the phase separation occurs mainly in the depth direction, but when the dewetting occurs at around 60 min after the temperature jump the phase separation is not completed. After the dewetting into the droplets the 
phase separation may proceed within the droplets. Outer surface of the droplets must be PVME because the preferential interactions between PVME and air [32-36]. The phase separation further proceeds and the surface PVME increases in thickness. Finally PVME comes out from the surface of the droplets onto the substrate to form a very thin layer $10 \mathrm{~nm}$ thick. Similar thin film formation was reported by Stamm et al [10] after a very long annealing of dPS and PpMS blend thin film.

\section{Conclusion}

In this study we investigated structure development of blend thin films of dPS (PS) and PVME below $\sim 200 \mathrm{~nm}$ at $115^{\circ} \mathrm{C}$ in the two phase region during the incubation period before the dewetting. It was found in the NR measurements that the thin film had a tri-layer structure consisting of the surface PVME layer, the middle blend layer and the bottom PVME layer even in the one phase region. This must be due to preferable interactions of PVME and air and PVME and Si substrate. When the thin

film was annealed at $115^{\circ} \mathrm{C}$ the interfacial roughness $\sigma_{\mathrm{NR} \text {,inter }}$ between the top PVME and the middle blend layer rapidly increased with the annealing time. This is the phase separation of the middle layer enhanced by the preferable interaction between PVME and air. This observation supported the dewetting mechanism induced by composition fluctuations. After very long annealing of $360 \mathrm{~min}$ the NR result clearly showed that a thin PVME layer was formed on the Si substrate. It implies that the phase separation proceeds in the dewetted droplets, and the inner PVME comes out, and finally wets the Si surface.

\section{References}


[1] Jones R L, Richards R W. Polymers at Surface and Interfaces. Cambridge: Cambridge University Press; 1999.

[2] Karim A, Kumar S. Polymer Surfaces, Interfaces and Thin Films. Singapore: World Scientific; 2000.

[3] Bucknall D G. Progress in Materials Science 2004; 49: 713-86.

[4] Mueller-Buschbaum P, Bauer E, Wunnicke O, Stamm M. J. Phys.: Condens. Matter 2005; 17: S363-86.

[5] Keddie J L, Jones R A, Cory R A. Europhys. Lett. 1994; 27: 59-64.

[6] Keddie J L, Jones R A, Cory R A. Faraday Discuss. 1994; 98: 219-30.

[7] Forrest J A, Dalnoki-Veress K, Stevens J R, Dutcher J R. Phys. Rev. Lett. 1996; 77: 2002-5.

[8] Kawana S, Jones R A L. Phys. Rev. E 2001; 63: 021501.

[9] Miyazaki T, Nishida K, Kanaya T. Phys. Rev. E 2004; 69: 06183-8.

[10] Mueller-Buschbaum P, O'Neil S A, Affrossman S, Stamm M. Macromolecules 1998; 31: 5003-9.

[11] Chung H, Composto R J. Phys. Rev. Lett. 2004; 92: 185704.

[12] Wang H, Composto R J. Europhys. Lett. 2000; 50: 622-7.

[13] Wang H, Composto R J. Chem. Phys. 2000; 113: 10386-97.

[14] Wang H, Composto R J. Interface Science 2003; 11: 237-48.

[15] Liao Y, Su Z, Sun Z, Shi T, An L. Macromol. Rapid Commun. 2006; 27: 351-5.

[16] Tanaka K, Yoon J S, Takahara A, Kajiyama T. Macromolecules 1995; 28: 934-8.

[17] Ermi B D, Karim A, Douglas J F. J. Poly. Sci., Part. B 1998; 36: 191-200.

[18] Karim A, Slawecki T M, Kumar S K, Douglas J F, Satija S K, Han C C, Russell T P, Liu Y, Overnay R, Sokolov J, Rafailovich M H. Macromolecules 1998; 857-62. 
[19] El-Mabrouk K, Belaiche M, Bousmina M. J. Coll. Interface Sci. 2007; 306: 354-

67.

[20] Chung H j, Ohno K, Fukuda T, Composto T J. Macromolecules 2007; 40: 384-8.

[21] Wensink K D F, Je'remoe B. Langmuir 2002; 18: 413-6.

[22] Sharma A, Mittal J. Phys. Rev. Lett. 2002; 89: 1861.

[23] Sharma A, Mittal J, Verma R. Langmuir 2002; 18: 10213-20.

[24] Clarke N. Macromolecules 2005; 38: 6775-8.

[25] Ogawa H, Kanaya T, Nishida K, Matsuba G. Polymer 2008; 40: 254-62.

[26] Nishida K, Kanaya T, Matsuba G, Ogawa H, Konishi T. Japanese Patent Pending $2005 ; 2005-058211$.

[27] Ebisawa T, Tasaki S, Otake Y, Funahashi H, Soyama K, Torikai N, Matsushita Y. Physica 1995; B213/214: 901-3.

[28] Braun C. Parratt32 program (Berlin: Berlin Neutron Scattering Center (BENSC), Hahn-Meitner Institut) .

[29] Parratt L G. Phys. Rev. 1954; 95: 359-69.

[30] Cahn J W, Hilliard J E. J. Chem. Phys. 1958; 28: 258-67.

[31] Cahn J W. J. Chem. Phys. 1965; 42: 93-9.

[32] Bhatia Q, Pan D, Koberstein J. Macromolecules 1988; 21: 2166-75.

[33] Cowie J, Devlin B, McEwen I. Macromolecules 1993; 26: 5628-32.

[34] Lee S, Sung C. Macromolecules 2001; 34: 599-604.

[35] Forrey C, Koberstein J, Pan D H. Interface Science 2003; 11: 211-23.

[36] Kawaguchi D, Tanaka K, Kajiyama T, Takahara A, Tasaki S. Macromolecules 2003; 36: 6824-30. 
[37] Jones R A L, Norton L J, Kramer E J, F S Bates, Wiltzius P, Phys. Rev. Lett. 1991; 66: 1326-29.

[38] Shull K R, Mayers A M, Russell T P. Macromolecules 1993; 26: 3929-36. 


\section{Figure Captions}

Figure 1. OM (a) and AFM (b) images of PS/PVME blend thin films after temperature jump to $115^{\circ} \mathrm{C}$ in the two phase region for four thickness $d$ regions. Region I (15 $\mu \mathrm{m}$ $<d)$ : $65 \mu \mathrm{m}, \quad$ Region II $(1 \mu \mathrm{m}<d<15 \mu \mathrm{m}): 2 \mu \mathrm{m}, \quad$ Region III $(200 \mathrm{~nm}<d<1 \mu \mathrm{m})$ : $466 \mathrm{~nm}$, Region IV $(d<200 \mathrm{~nm}): 98 \mathrm{~nm}$ and time evolution of LS profiles (c) after temperature jump to $115^{\circ} \mathrm{C}$ in the two phase region. Scale bars in $\mathrm{OM}$ images are 20 $\mu \mathrm{m}$. Z-range is $2.0 \mu \mathrm{m}$ and $\mathrm{x}-\mathrm{y}$ size is $25 \mu \mathrm{m} \times 25 \mu \mathrm{m}$ for all AFM images.

Figure 2. Time evolution of OM images of PS/PVME blend film $98 \mathrm{~nm}$ thick after temperature jump to $115^{\circ} \mathrm{C}$ in the two phase region. Scale bars in $\mathrm{OM}$ images are 20 $\mu \mathrm{m}$.

Figure 3. Time evolution of peak position $q_{\max }$ and peak intensity $I_{\max }$ in the LS profile of PS/PVME blend film $98 \mathrm{~nm}$ thick after temperature jump to $115^{\circ} \mathrm{C}$ in the two phase region. Peak intensity $I_{\max }$ in the incubation period before $\sim 30$ min is intensity at $q=2$ $\mu \mathrm{m}^{-1}$.

Figure 4. NR profiles for dPS/PVME blend films 180, 98, 42 and $25 \mathrm{~nm}$ thick at $25^{\circ} \mathrm{C}$ in the one phase region and the results of fits (solid curves) with the tri-layer model. Insets show scattering length density (SLD) profiles in depth direction evaluated in the NR profile fits. 
Figure 5. (a): Time evolution of NR profile for dPS/PVME blend film $98 \mathrm{~nm}$ thick annealed at $115^{\circ} \mathrm{C}$ in the two phase region. Measurements were done after quenching to room temperature. The solid curves are the results of fits with the tri-layer model. (b): Scattering length density (SLD) profiles in depth direction as a function of annealing time.

Figure 6. Time evolutions of interface roughness $\sigma_{\mathrm{NR}, \text { inter }}$ evaluated from the NR data and the surface roughness $\sigma_{\mathrm{AFM} \text {,surf }}$ from AFM data, respectively, for dPS/PVME blend film $98 \mathrm{~nm}$ thick annealed at $115^{\circ} \mathrm{C}$ in the two phase region.

Figure 7. Time evolution of AFM images of dPS/PVME blend film $98 \mathrm{~nm}$ thick after temperature jump to $115^{\circ} \mathrm{C}$ in the two phase region. Scale bars in AFM images are 5 $\mu \mathrm{m}$.

Figure 8. Distribution $f(h)$ of surface heights in the surface of dPS/PVME film $98 \mathrm{~nm}$ thick annealed at $115^{\circ} \mathrm{C}$ for $5 \mathrm{~min}$. The solid curve is result of fit to a Gaussian function. 
(a)

(b)

Region I $(15 \mu \mathrm{m}<d)$
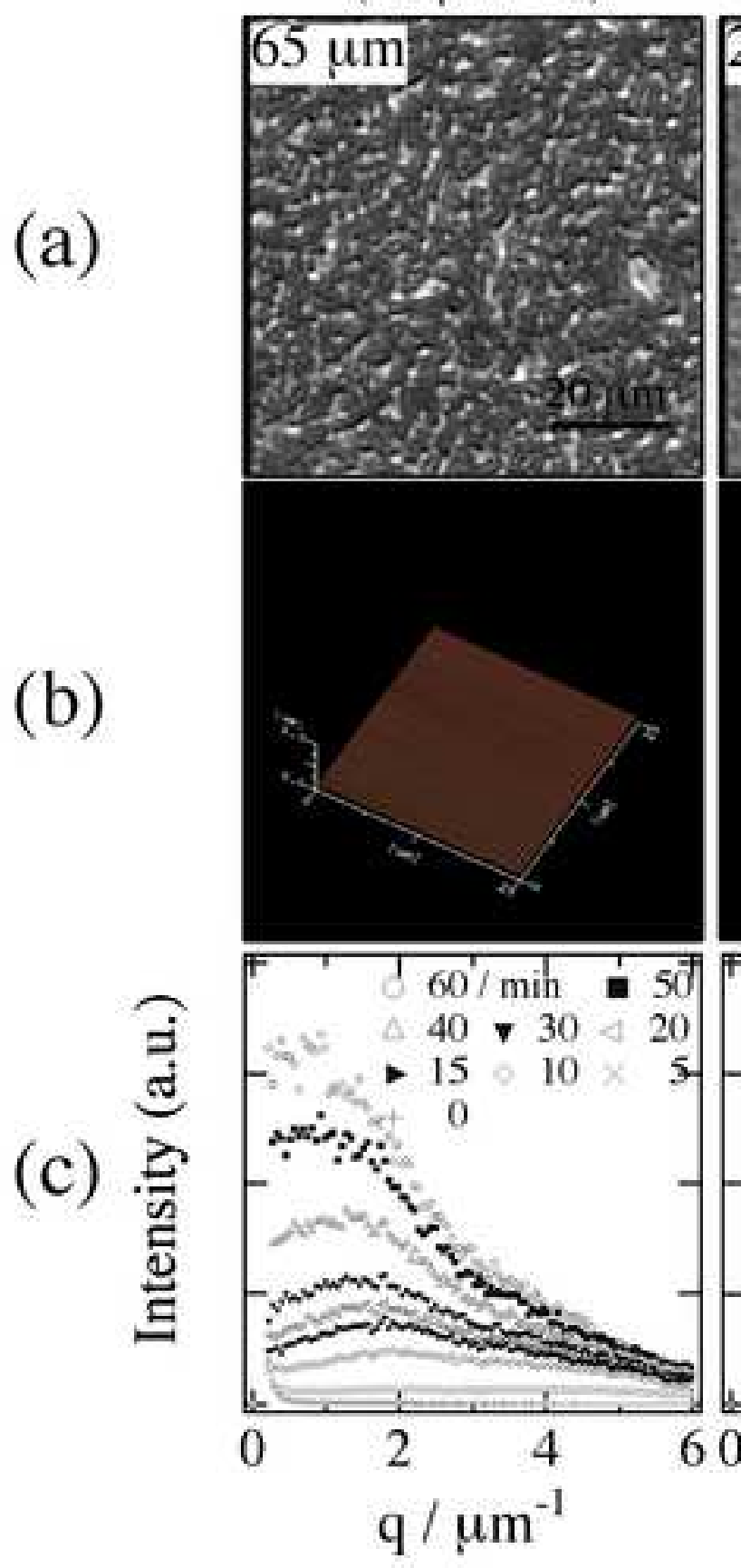

Region II $(1 \mu \mathrm{m}<d<15 \mu \mathrm{m})(200 \mathrm{~nm}<d<1 \mu \mathrm{m})$
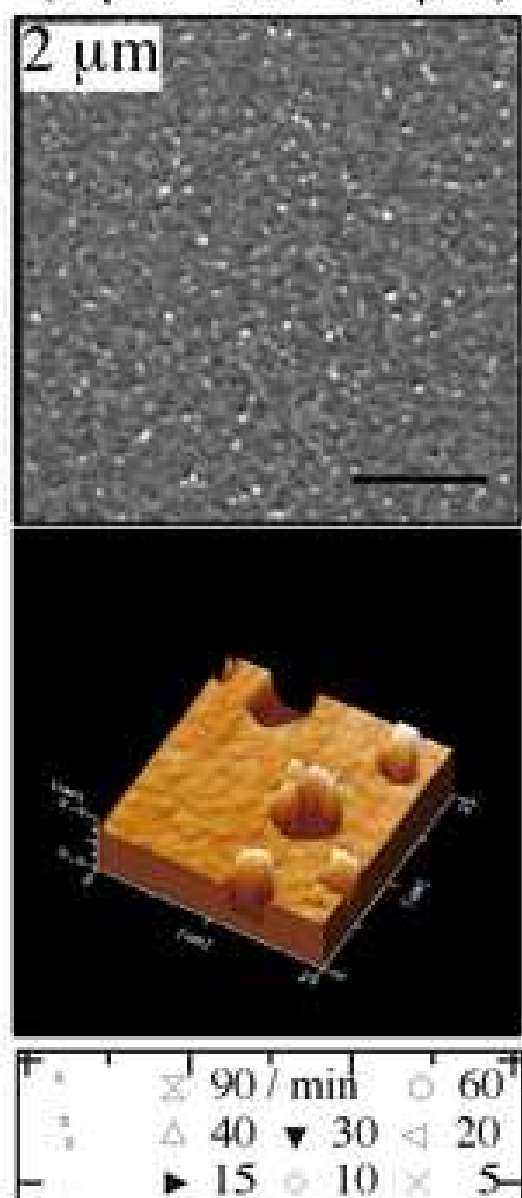

$-15 \quad 10-5$
0
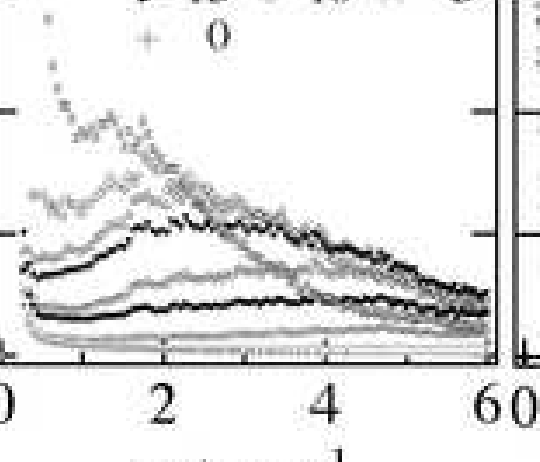

$\mathrm{q} / \mu \mathrm{m}^{-1}$

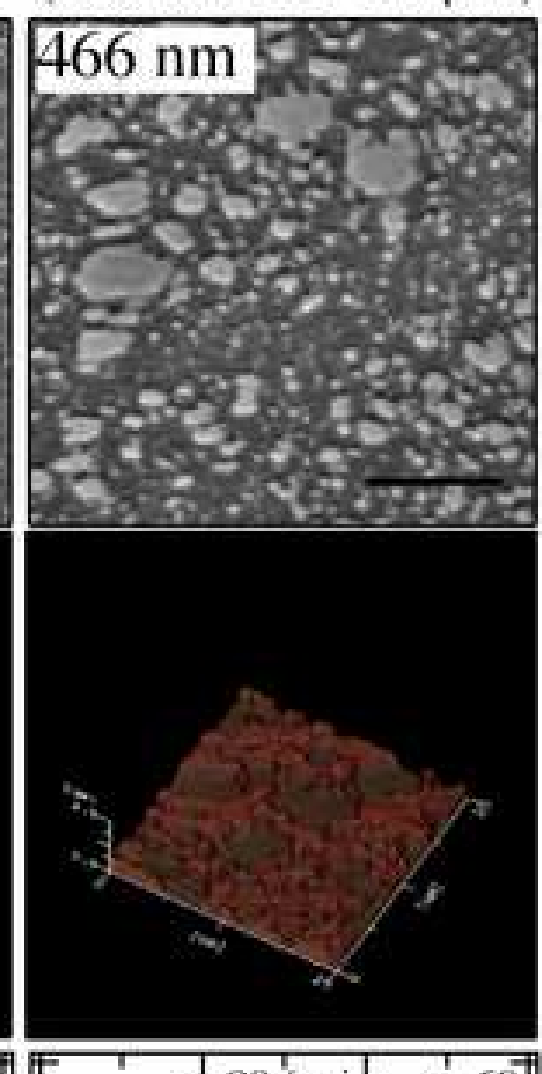

Region III

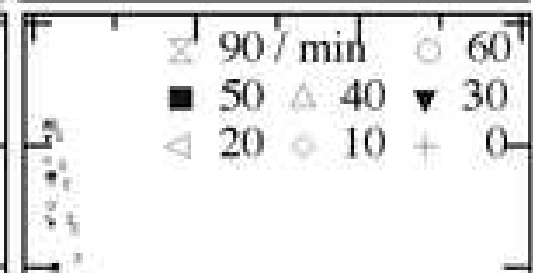

Region IV $(d<200 \mathrm{~nm})$
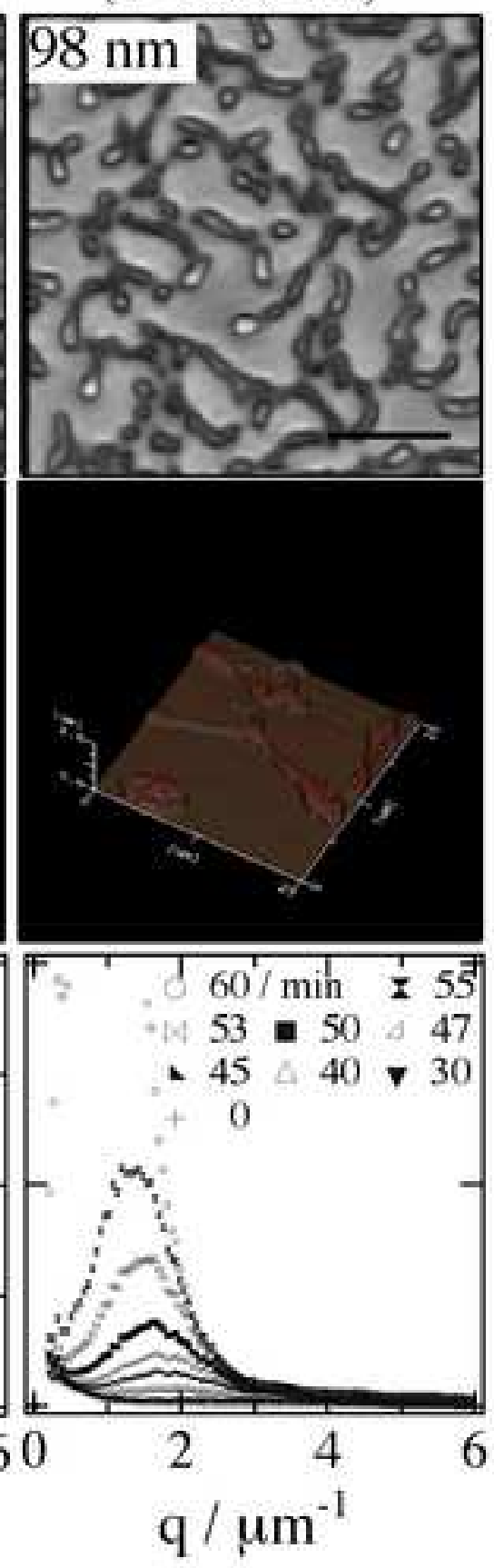


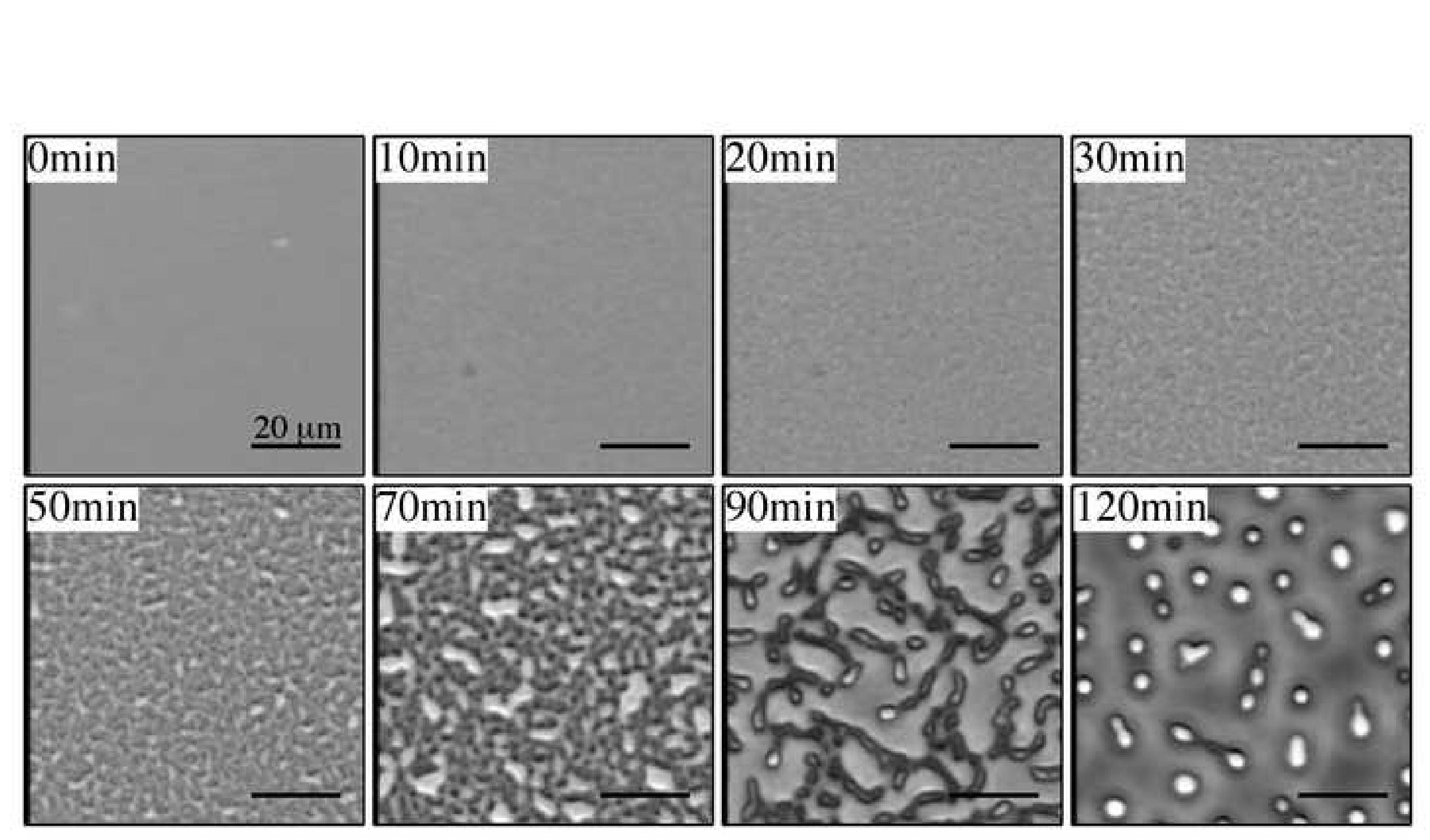

to download high resolution image 


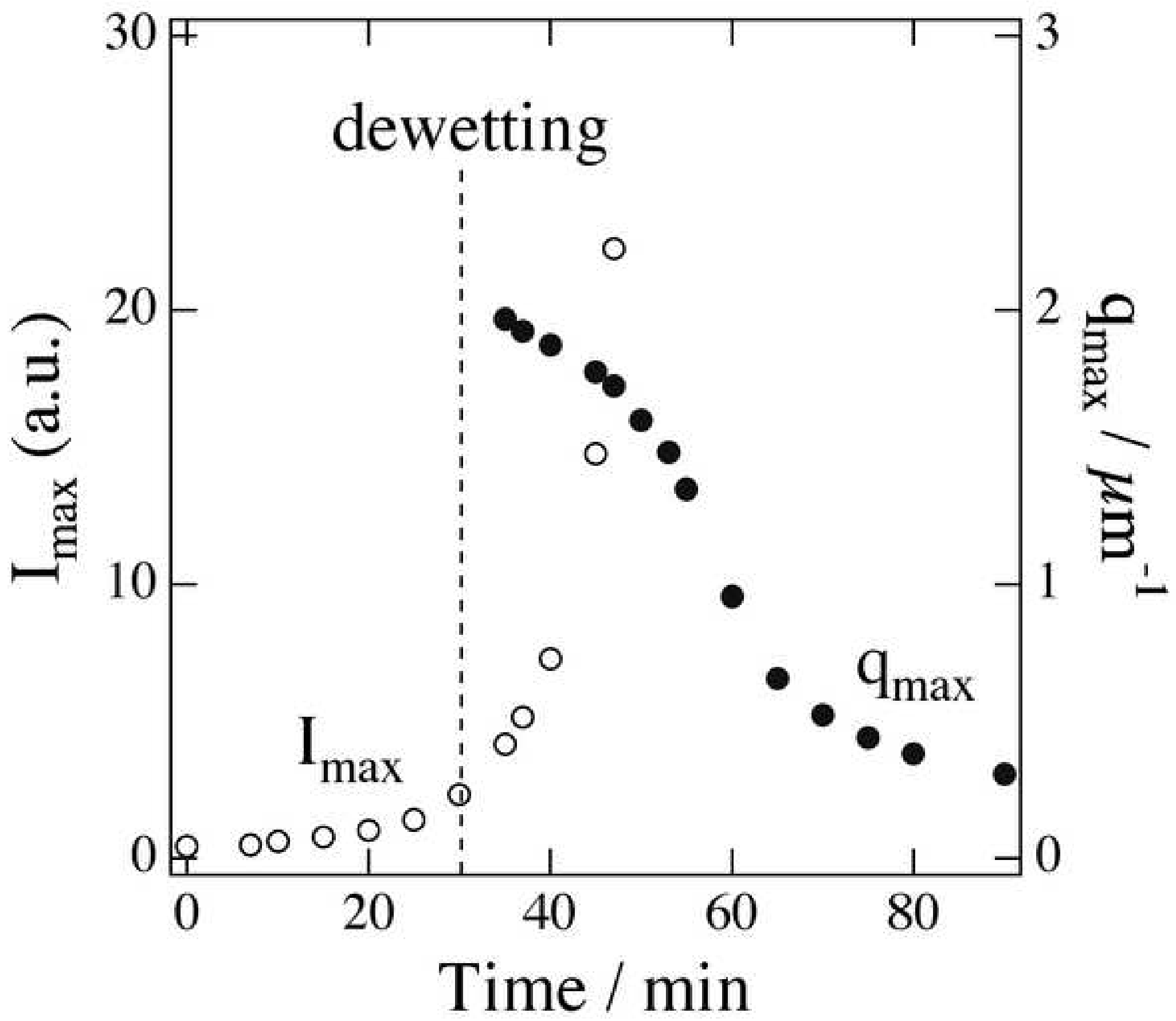




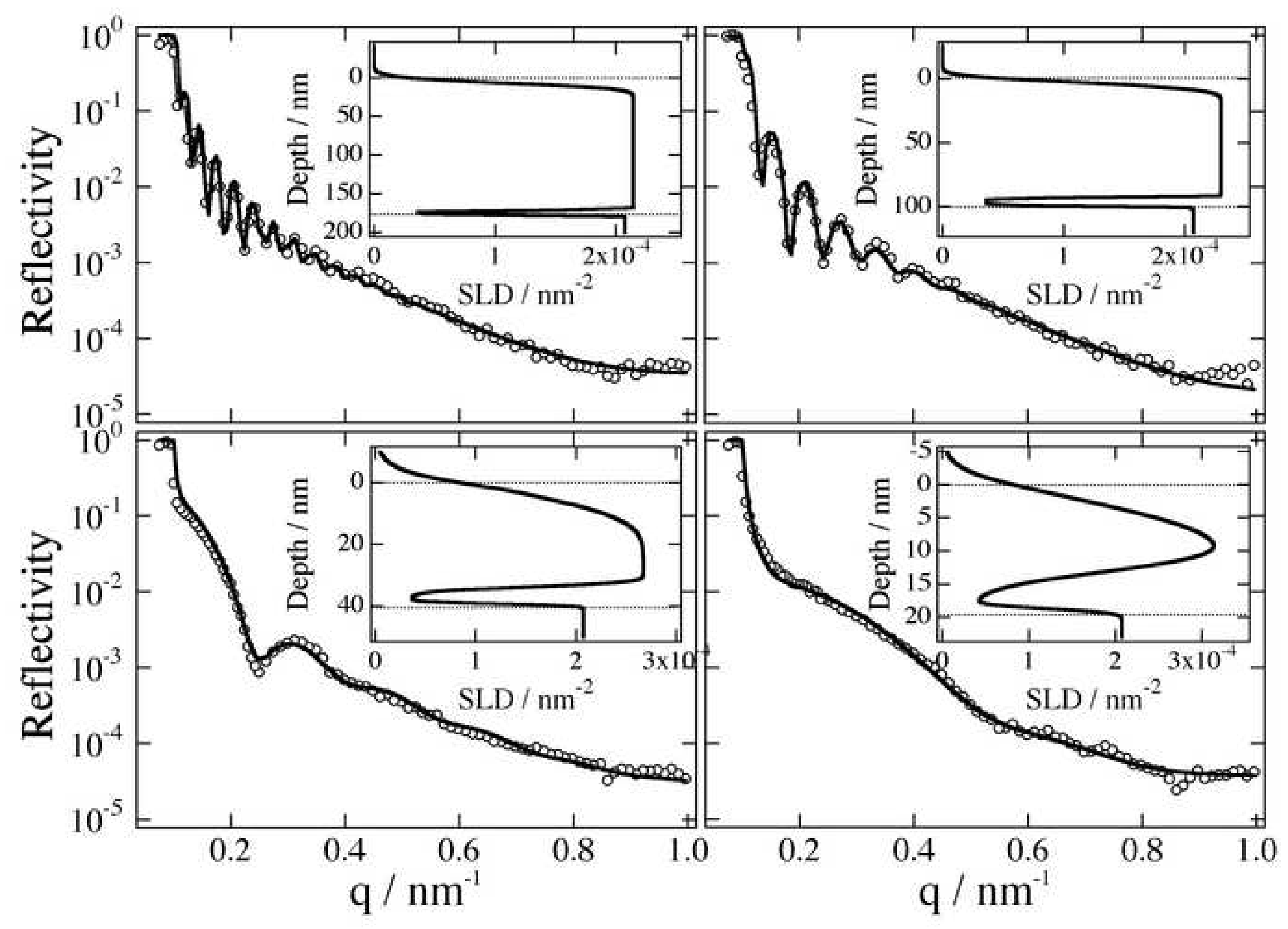

(1)



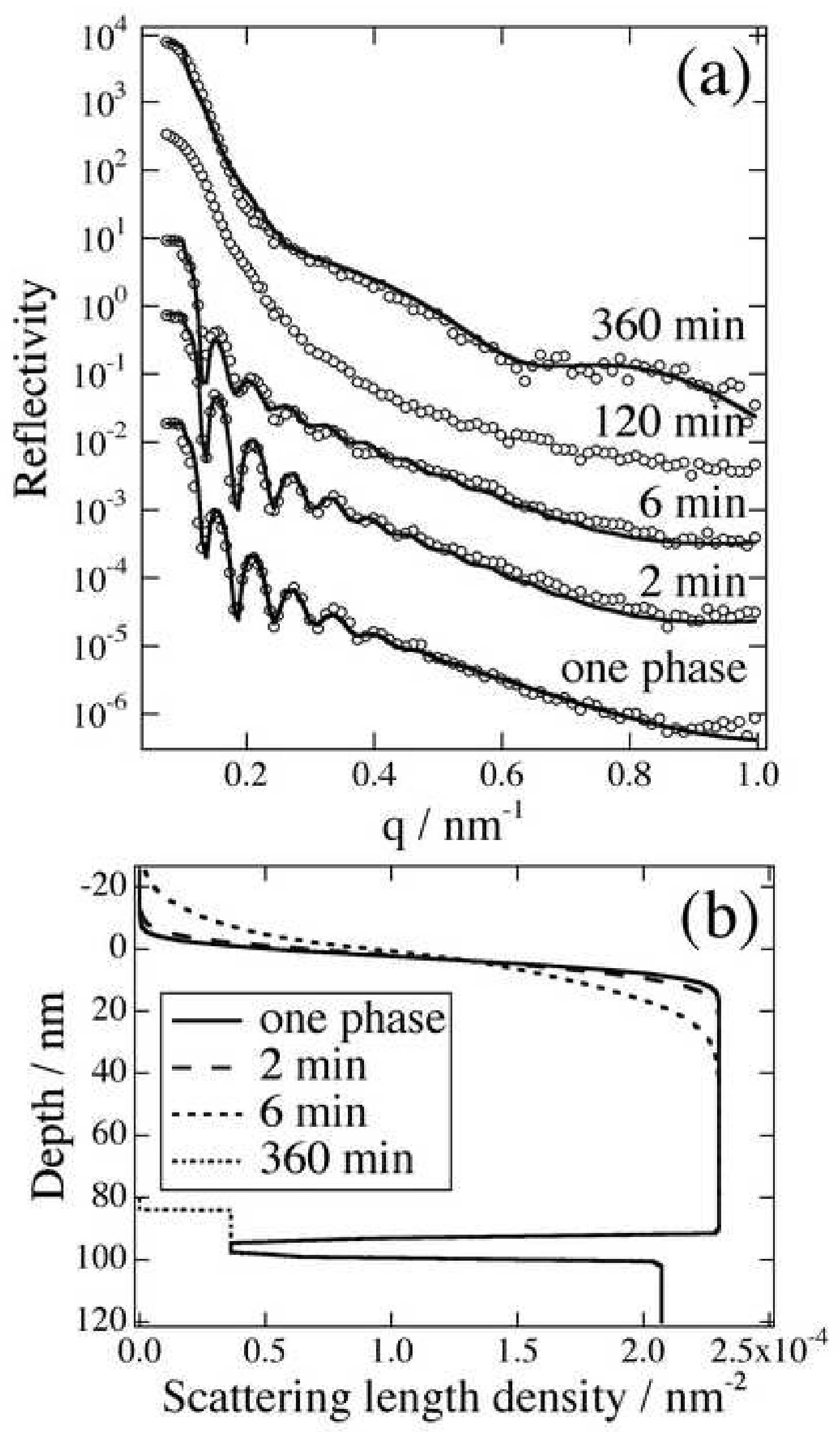

\section{$\underline{I}$}




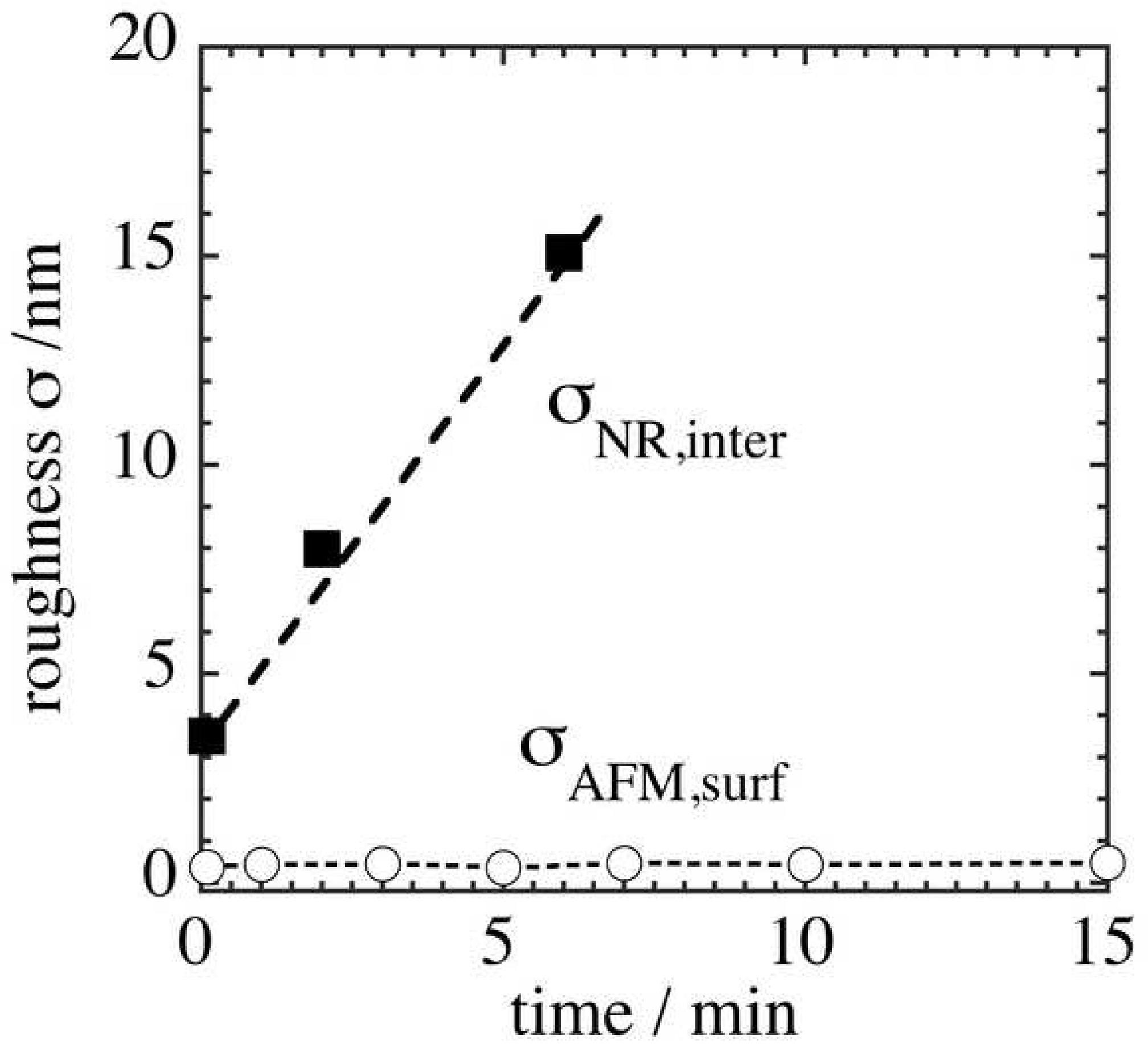



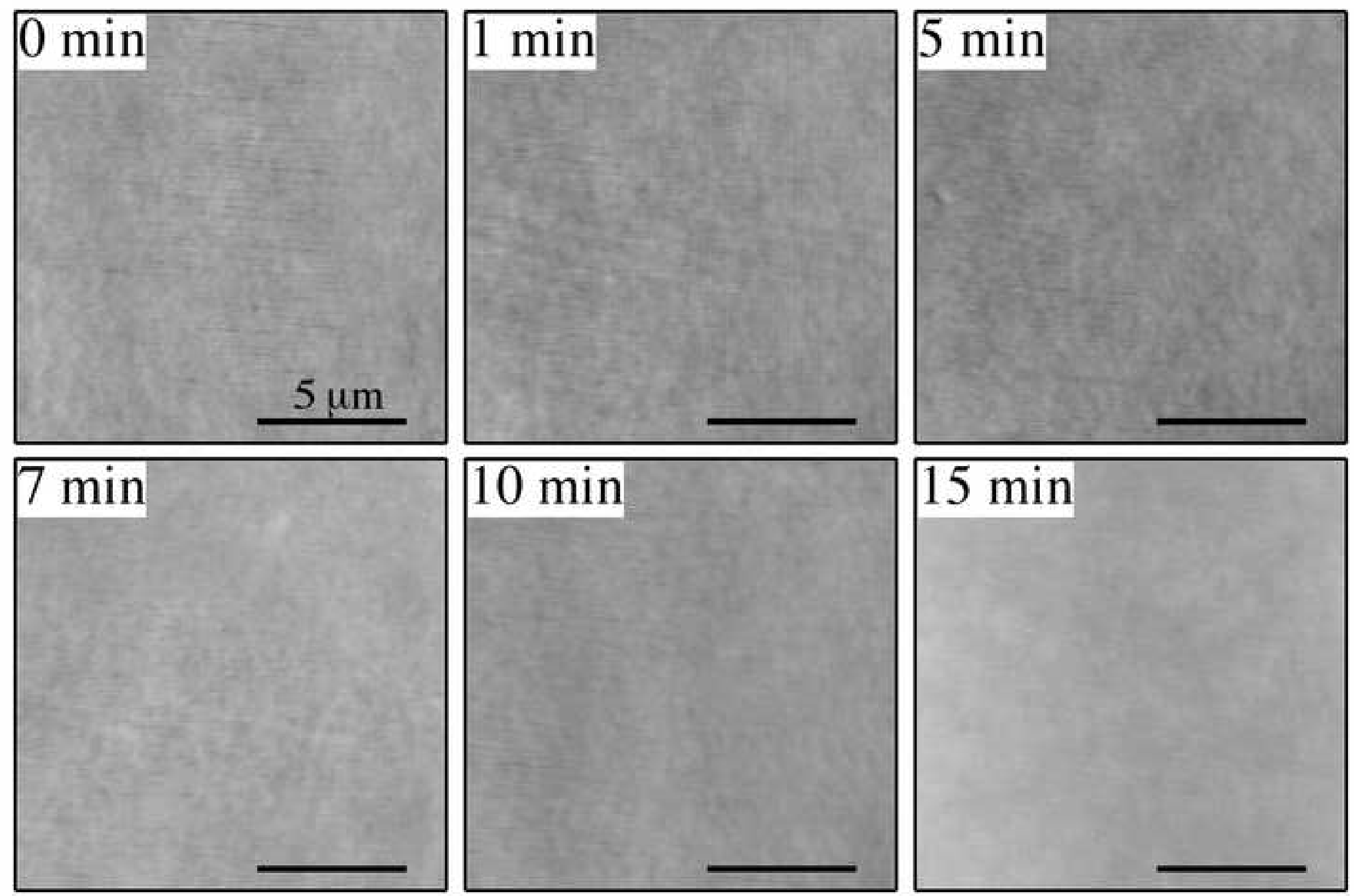

$15 \mathrm{~min}$

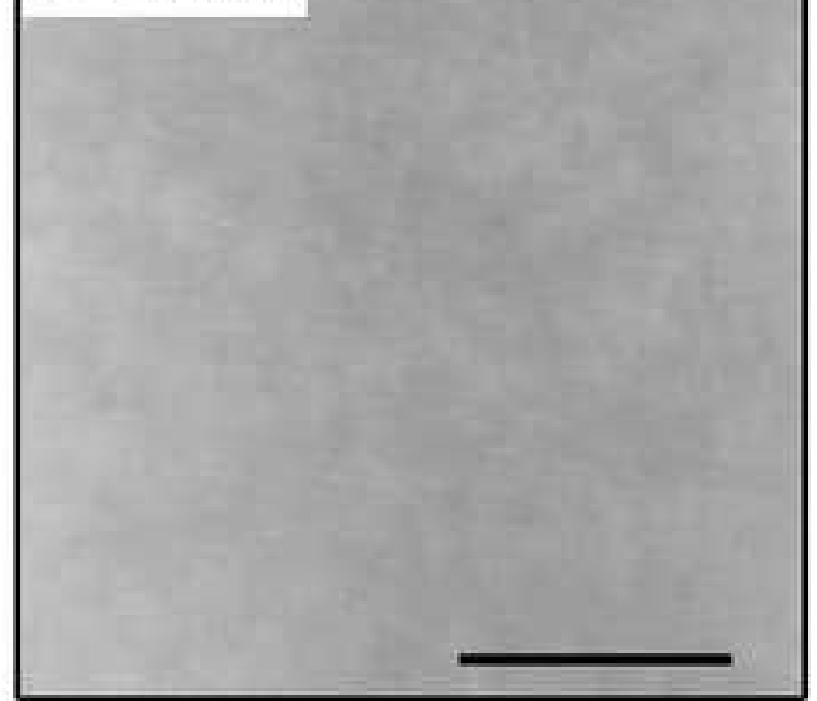




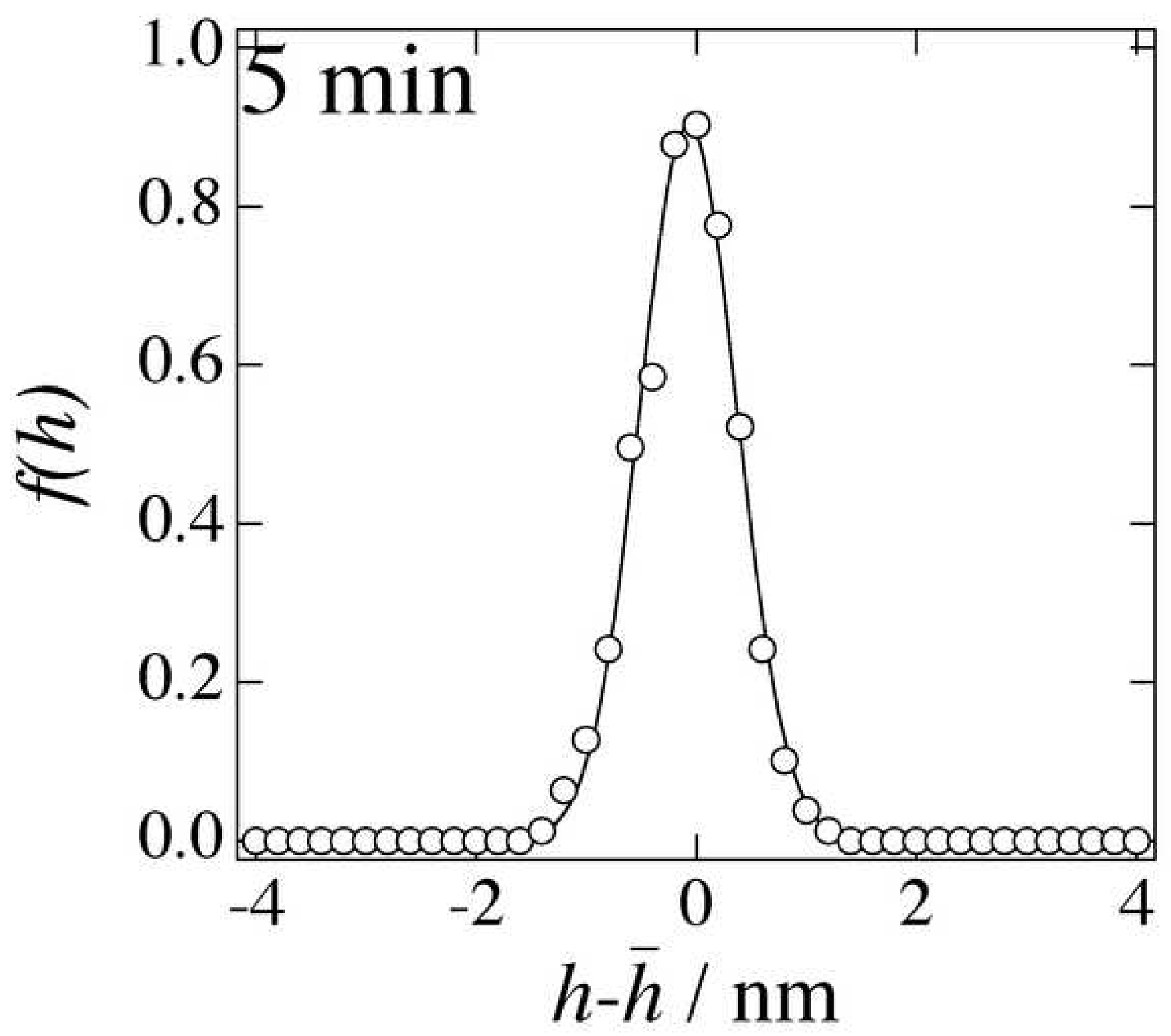

